\title{
MULTIPLE SOLUTIONS FOR COMPRESSIBLE TURBULENT FLOW MODELS
}

\author{
CHRISTOPHE BERTHON ${ }^{\dagger}$ AND FRÉDÉRIC COQUEL CH $^{\ddagger}$
}

\begin{abstract}
We analyse the uniqueness of the solutions of a PDE system from the framework of compressible turbulent models. Smoothness properties of the turbulent viscosity closure law are of central importance. Several closures of practical importance, including the most widely used law, indeed fail to be Lipschitz continuous in the natural neighborhood of a null turbulent energy. For such models, we prove the existence of infinitely many distinct traveling wave solutions which exhibit positive turbulent energy but connect at infinity end states with vanishing turbulence. Examples and counter-examples are given.
\end{abstract}

Key words. Traveling wave solutions, non-uniqueness, compressible gas dynamics systems

AMS subject classifications. 35L67, 35Q35, 76F50

\section{Introduction}

The present work examines uniqueness properties of the solutions of a PDE model directly motivated by the physics of compressible turbulent flows. The system under consideration naturally writes as an extension of the usual Navier-Stokes equations involving an additional PDE for governing a supplementary internal energy. This socalled turbulent energy dictates a turbulent viscosity law which sum with the laminar one defines the total viscosity function. A relaxation term drives this turbulent energy to vanish in time, in agreement with the physics. The key issue comes from the property that most of the turbulent viscosity laws, proposed in the literature, fail to be Lipschitz continuous in the regime of a vanishing turbulent energy. Such a lack of smoothness therefore rises the question of uniqueness of natural solutions of the PDE model which exhibit locally zero turbulent energy. We answer this question when studying both existence and uniqueness of the traveling wave solutions of the system connecting end states with null turbulent energy. Due to the interplay between the relaxation term and the turbulent viscosity law, the reported lack of smoothness is proved to result in infinitely many distinct traveling wave solutions connecting the same pair of end states at infinity. We underline that failure of uniqueness applies to the most widely used turbulent viscosity closure. Up to our knowledge, this negative but important result seems to be new. By contrast, we report a closure law, proposed in the literature, for which existence and uniqueness are guaranteed.

The format of the present paper is the following. The first section describes the mathematical model with a special attention paid on the precise properties met by both turbulent viscosity law and relaxation source term. We emphasize that the proposed assumptions are indeed satisfied by the models proposed in the literature. The main result of the present paper is then stated. The second section is devoted to the analysis of the traveling solutions of the PDE system. Multiple solutions are built

\footnotetext{
*Received: January 16, 2006; accepted (in revised version): May 19, 2006. Communicated by Lorenzo Pareschi.

${ }^{\dagger}$ MAB, UMR 5466, LRC M03, Université Bordeaux 1, 351 cours de la libération, 33400 Talence, France. INRIA Futurs, projet ScAlApplix, Domaine de Voluceau-Rocquencourt, B. P. 105, 78153 Le Chesnay Cedex, France.

${ }^{\ddagger}$ CNRS and Laboratoire Jacques Louis Lions, URA 189, Tour 55-65, Université Paris VI, 4 Place Jussieu, 75252 Paris, France.
} 
from the study of a suitable auxiliary problem. The last paragraph gives examples taken from the literature.

\section{Mathematical model}

The present paper aims at studying the existence and uniqueness of the traveling wave solutions of the following PDE model:

$$
\left\{\begin{array}{l}
\partial_{t} \rho+\partial_{x} \rho u=0, \quad x \in \mathbb{R}, t>0, \\
\partial_{t} \rho u+\partial_{x}\left(\rho u^{2}+p_{1}+p_{2}\right)=\partial_{x}\left(\left(\mu_{1}\left(\rho, T_{1}\right)+\mu_{2}\left(\rho, T_{2}\right)\right) \partial_{x} u\right), \\
\partial_{t} p_{1}+\partial_{x} p_{1} u+\left(\gamma_{1}-1\right) p_{1} \partial_{x} u=\left(\gamma_{1}-1\right) \mu_{1}\left(\rho, T_{1}\right)\left(\partial_{x} u\right)^{2}+\left(\gamma_{1}-1\right) \rho \epsilon\left(T_{2}\right), \\
\partial_{t} p_{2}+\partial_{x} p_{2} u+\left(\gamma_{2}-1\right) p_{2} \partial_{x} u=\left(\gamma_{2}-1\right) \mu_{2}\left(\rho, T_{2}\right)\left(\partial_{x} u\right)^{2}-\left(\gamma_{2}-1\right) \rho \epsilon\left(T_{2}\right) .
\end{array}\right.
$$

This model stands as a natural extension of the classical Navier-Stokes equations for a compressible gas with density $\rho>0$ and velocity $u \in \mathbb{R}$ obtained when considering two independent pressure laws $p_{1}$ and $p_{2}$ governed by their own PDE. Here the adiabatic coefficients $\gamma_{1}$ and $\gamma_{2}$ are two given real constants satisfying

$$
1<\gamma_{1}<\gamma_{2}
$$

Then the temperatures $T_{i}$, supposed to stay non-negative, are defined from $p_{i}=\left(\gamma_{i}-\right.$ 1) $\rho T_{i}$ with $i=1,2$. Next, the source term in (2.1) is assumed at this stage to satisfy:

$$
\begin{array}{rll}
\epsilon\left(T_{2}\right)>0 \text { for all } T_{2}>0 \text { with } & \lim _{T_{2} \rightarrow 0^{+}} \epsilon\left(T_{2}\right)=0, \\
\epsilon \in \mathcal{C}^{1}\left(\mathbb{R}^{+}, \mathbb{R}^{+}\right) \text {with } & \lim _{T_{2} \rightarrow 0^{+}} \frac{d}{d T_{2}} \epsilon\left(T_{2}\right)=0 .
\end{array}
$$

This source term intends to relax the pressure $p_{2}$ to zero as the time goes to infinity in view of the ODE

$$
d_{t} p_{2}=\frac{\epsilon\left(T_{2}\right)}{T_{2}}\left(0-p_{2}\right)
$$

inferred from (2.1) when neglecting the space derivatives. These considerations lead us to introduce the following natural phase space for (2.1):

$$
\Omega=\left\{\mathbf{v}={ }^{t}\left(\rho, \rho u, p_{1}, p_{2}\right) \in \mathbb{R}^{4} / \rho>0, \rho u \in \mathbb{R}, p_{1}>0, p_{2} \geq 0\right\} .
$$

At last, the required viscosity laws are such that

$$
\mu_{1}\left(\rho, T_{1}\right)+\mu_{2}\left(\rho, T_{2}\right)>0, \quad \text { for all } \mathbf{v} \in \Omega,
$$

and their precise forms depend on the physical setting. Models from the physics of compressible turbulent flows enter in particular the present framework where $\mu_{1}\left(\rho, T_{1}\right)$ is the so-called laminar viscosity while $\mu_{2}\left(\rho, T_{2}\right)$ represents the turbulent viscosity function (see $[1,2,3]$ for the details). Let us just mention that $T_{2}$ coincides with the specific kinetic turbulent energy usually denoted by $k$ with the adiabatic coefficient given by $\gamma_{2}=5 / 3$. Here the relaxation term $\epsilon(k)=\mathcal{L} k^{3 / 2}, \mathcal{L}>0$, is the turbulent dissipation rate proposed in the so-called mixing length model where $\mathcal{L}$ denotes the inverse of the mixing length (see for instance Mohammadi-Pironneau [10] or Hirsh [7] and the references therein).

Motivated by several models (see section 4 for examples of interest) the viscosity laws in the present work will be assumed to satisfy two distinct sets of smoothness 
properties. The first setting for viscosity laws in the form $\mu(\rho, y) \geq 0$ with $(\rho, y) \in$ $\mathbb{R}_{\star}^{+} \times \mathbb{R}^{+}$is such that

$$
\mu \in \mathcal{C}^{1}\left(\mathbb{R}_{\star}^{+} \times \mathbb{R}^{+}, \mathbb{R}^{+}\right) .
$$

Throughout this paper, the viscosity law $\mu_{1}\left(\rho, T_{1}\right)$ is assumed to obey (2.6). By contrast, the second law $\mu_{2}\left(\rho, T_{2}\right)$ may or not satisfy this smoothness condition. An important and general property, grounded from the physics, requires the viscosity law $\mu_{2}\left(\rho, T_{2}\right)$ to vanish with $T_{2}$; namely:

$$
\lim _{y \rightarrow 0^{+}} \mu_{2}(\rho, y)=0, \quad \rho>0 .
$$

All the turbulent models proposed in the literature $[7,10,11]$ are such that the mapping $(\rho, y) \in \mathbb{R}_{\star}^{+} \times \mathbb{R}_{\star}^{+} \rightarrow \mu_{2}(\rho, y) \in \mathbb{R}^{+}$is differentiable but for most of them, this smoothness property does not extend to $(\rho, y) \in \mathbb{R}_{\star}^{+} \times \mathbb{R}^{+}$: namely, $\rho>0$ being fixed, their typical asymptotic behavior, for $y>0$ small enough, is in $\mathcal{O}\left(y^{\alpha}\right)$ for some $\alpha \in[1 / 2,1)$, so that Lipschitz continuity is lost when $y$ vanishes. It turns out that such models which fail to obey (2.6) satisfy by contrast the following three properties:

$$
\lim _{y \rightarrow 0^{+}} \frac{\mu_{2}(\rho, y)}{y^{\alpha}}=h(\rho)>0, \quad \rho>0,
$$

with the property that the function:

$$
\begin{aligned}
& (\rho, z) \in(0,+\infty) \times[0,+\infty) \rightarrow \Phi(\rho, z)=\mu_{2}\left(\rho, z^{\frac{1}{1-\alpha}}\right) \in \mathbb{R}^{+} \\
& \text {is Lipschitz continuous, }
\end{aligned}
$$

together with:

$$
\begin{aligned}
& (\rho, z) \in(0,+\infty) \times[0,+\infty) \rightarrow \Psi(\rho, z)=\frac{\mu_{2}\left(\rho, z^{\frac{1}{\alpha-\alpha}}\right)}{z^{\frac{\alpha}{1-\alpha}}} \in \mathbb{R}^{+} \\
& \text {is Lipschitz continuous. }
\end{aligned}
$$

Motivated by the physics [11] and the setting of assumptions (2.8)-(2.10) for the second viscosity law, the relaxation source term $y \in \mathbb{R}^{+} \rightarrow \epsilon(y) \in \mathbb{R}^{+}$in (2.1), is assumed to obey the following strengthened conditions:

$$
\begin{aligned}
& \epsilon(y)>0 \quad \text { for all } y>0 \quad \text { with } \lim _{y \rightarrow 0^{+}} \frac{\epsilon(y)}{y^{\alpha}}=0, \\
& y \in \mathbb{R}^{+} \rightarrow \frac{\epsilon(y)}{y^{\alpha}} \in \mathbb{R}^{+} \quad \text { is Lipschitz continuous }
\end{aligned}
$$

where $\alpha$ is the exponent used in (2.8)-(2.10).

Let us stress that the reported properties are in particular met by the most widely used turbulent viscosity law $\mu_{2}(\rho, y)=C \rho y^{1 / 2}$ so that (2.7) to (2.10) hold with $\alpha=\frac{1}{2}$, while the turbulent dissipation rate $\epsilon(y)=\mathcal{L} y^{3 / 2}, \mathcal{L}>0$, satisfies (2.11) again with $\alpha=1 / 2$. We refer the reader to [11]. Other closure laws for the turbulent viscosity are examined at the end of the paper.

We claim uniqueness in the traveling wave solutions of the system (2.1), we rewrite for short

$$
\partial_{t} \mathbf{v}+\mathbf{A}(\mathbf{v}) \partial_{x} \mathbf{v}=\mathcal{R}\left(\mathbf{v}, \partial_{x x} \mathbf{v}\right)+\mathbf{S}(\mathbf{v}), \quad x \in \mathbb{R}, t>0
$$


is actually dictated by the smoothness properties met by the viscosity law $\mu_{2}\left(\rho, T_{2}\right)$, namely either (2.6) or (2.7)-(2.10). In order to state the main result of the present paper, we first briefly recall that a traveling wave solution of (2.1) is a smooth solution in $\mathrm{C}^{1}\left(\mathbb{R} \times \mathbb{R}^{+}, \Omega\right)$ of the form $\mathbf{v}(x, t)=\widehat{\mathbf{v}}(x-\sigma t)$ with $\sigma \in \mathbb{R}$ and

$$
\left\{\begin{array}{l}
\lim _{\xi \rightarrow-\infty} \widehat{\mathbf{v}}(\xi)=\mathbf{v}_{L}, \quad \lim _{\xi \rightarrow+\infty} \widehat{\mathbf{v}}(\xi)=\mathbf{v}_{R}, \quad \xi=x-\sigma t, \\
\lim _{\xi \rightarrow \pm \infty} \widehat{\mathbf{v}}^{\prime}(\xi)=\lim _{\xi \rightarrow \pm \infty} \widehat{\mathbf{v}}^{\prime \prime}(\xi)=0,
\end{array}\right.
$$

where $\left(\mathbf{v}_{L}, \mathbf{v}_{R}\right) \in \Omega^{2}$. This particular smooth solution must therefore satisfy the following nonlinear ODE's system:

$$
-\sigma d_{\xi} \widehat{\mathbf{v}}+\mathbf{A}(\widehat{\mathbf{v}}) d_{\xi} \widehat{\mathbf{v}}=\mathcal{R}\left(\widehat{\mathbf{v}}, d_{\xi \xi}^{2} \widehat{\mathbf{v}}\right)+\mathbf{S}(\widehat{\mathbf{v}}), \quad \xi \in \mathbb{R} .
$$

Let us first stress that the asymptotic conditions (2.13) can be achieved only if the two end states meet the following special form:

$$
\mathbf{v}_{L}=\left(\rho_{L},(\rho u)_{L},\left(p_{1}\right)_{L}, 0\right), \quad \mathbf{v}_{R}=\left(\rho_{R},(\rho u)_{R},\left(p_{1}\right)_{R}, 0\right),
$$

so as to force the source term $\rho \epsilon\left(T_{2}\right)$ in (3.6) to identically vanish at infinity (see indeed the general assumption (2.3)).

As it is well-known, traveling wave solutions can only be associated with genuinely nonlinear fields in the underlying first order system (see [13] for instance). This one is seen to be hyperbolic over the phase space $\Omega$ with the following distinct eigenvalues

$$
u-c(\mathbf{v}), \quad u, \quad u+c(\mathbf{v}), \quad c(\mathbf{v})=\sqrt{\frac{\gamma_{1} p_{1}+\gamma_{2} p_{2}}{\rho}}, \quad \mathbf{v} \in \Omega,
$$

where $u$ has two order of multiplicity. The extreme fields are genuinely nonlinear while the intermediate ones are linearly degenerate. For frame invariance properties satisfied by the PDE model (2.1), it suffices to study the traveling wave solutions associated with the first extreme field [13]. We are now in a position to state our main result:

Theorem 2.1. Let be given a state $\mathbf{v}_{L} \in \Omega$ with $\left(p_{2}\right)_{L}=0$. Let a velocity $\sigma$ be prescribed under the Lax condition:

$$
u_{L}-c\left(\mathbf{v}_{L}\right)>\sigma, \quad c^{2}\left(\mathbf{v}_{L}\right)=\frac{\gamma_{1}\left(p_{1}\right)_{L}}{\rho_{L}} .
$$

- Assume that both viscosity laws $\mu_{1}\left(\rho, T_{1}\right)$ and $\mu_{2}\left(\rho, T_{2}\right)$ achieve the smoothness property (2.6) while the relaxation term $\epsilon\left(T_{2}\right)$ obeys (2.3). Then there exists a unique (up to some translation) traveling wave solution issuing from $\mathbf{v}_{L}$ and arriving at a state $\mathbf{v}_{R}$ in $\Omega$ with $\left(p_{2}\right)_{R}=0$.

- Assume that $\mu_{1}\left(\rho, T_{1}\right)$ obeys (2.6) while by contrast $\mu_{2}\left(\rho, T_{2}\right)$ satisfies (2.7)(2.10) and $\epsilon\left(T_{2}\right)$ meets (2.11). Then there exists infinitely many distinct traveling wave solutions connecting $\mathbf{v}_{L}$ to an end point $\mathbf{v}_{R}$ with $\left(p_{2}\right)_{R}=0$.

In case of uniqueness, the pressure $p_{2}$ stays identically zero along the profile while by contrast it exhibits strictly positive values in the reported multiple solutions. Let us underline that in both cases, the end point $\mathbf{v}_{R}=\mathbf{v}_{R}\left(\sigma, \mathbf{v}_{L}\right)$ is the same. Its precise form is detailed in the next section. 


\section{Traveling wave analysis}

The analysis makes use of the following result:

Lemma 3.1. Smooth solutions of (2.1) satisfy the following conservation law:

$$
\partial_{t} \rho E(\mathbf{v})+\partial_{x}\left(\rho E(\mathbf{v})+p_{1}+p_{2}\right) u=\partial_{x}\left(\left(\mu_{1}\left(\rho, T_{1}\right)+\mu_{2}\left(\rho, T_{2}\right)\right) u \partial_{x} u\right),
$$

where the total energy $\rho E(\mathbf{v})$ is defined by:

$$
\rho E(\mathbf{v})=\frac{(\rho u)^{2}}{2 \rho}+\frac{p_{1}}{\gamma_{1}-1}+\frac{p_{2}}{\gamma_{2}-1} .
$$

Smooth solutions satisfy in addition the following balance equations:

$$
\begin{aligned}
& \partial_{t} \rho s_{1}(\mathbf{v})+\partial_{x} \rho s_{1}(\mathbf{v}) u=\frac{\gamma_{1}-1}{\rho^{\gamma_{1}-1}}\left(\mu_{1}\left(\rho, T_{1}\right)\left(\partial_{x} u\right)^{2}+\rho \epsilon\left(T_{2}\right)\right), \\
& \partial_{t} \rho s_{2}(\mathbf{v})+\partial_{x} \rho s_{2}(\mathbf{v}) u=\frac{\gamma_{2}-1}{\rho^{\gamma_{2}-1}}\left(\mu_{2}\left(\rho, T_{2}\right)\left(\partial_{x} u\right)^{2}-\rho \epsilon\left(T_{2}\right)\right),
\end{aligned}
$$

where the specific entropies are respectively given by

$$
s_{1}(\mathbf{v}):=\frac{p_{1}}{\rho^{\gamma_{1}}}, \quad s_{2}(\mathbf{v}):=\frac{p_{2}}{\rho^{\gamma_{2}}} .
$$

The proof of the above result is easy and is left to the reader (see [1] and also [5] for the usual gas dynamics setting). The interest in Lemma 3.1 comes from the property that the mappings $\left(\rho, \rho u, p_{1}, p_{2}\right) \rightarrow\left(\rho, \rho u, \rho E, p_{2}\right)$ and $\left(\rho, \rho u, p_{1}, p_{2}\right) \rightarrow\left(\rho, \rho u, \rho s_{1}, \rho s_{2}\right)$, clearly define admissible changes of variables which turn out to be useful in the sequel.

3.1. The dynamical system. This paragraph aims at introducing the dynamical system which governs the traveling wave solutions we analyse for existence. Basic properties of the flow are stated.

Due to Galilean invariance (see $[4,14])$, it suffices to consider the case of a null velocity $\sigma$ in (2.14) when invoking the change of variables $\left(\rho, \rho u, p_{1}, p_{2}\right) \rightarrow\left(\rho, \rho u, \rho E, p_{2}\right)$ :

$$
\left\{\begin{array}{l}
d_{\xi}(\rho u)=0, \\
d_{\xi}\left(\rho u^{2}+p_{1}+p_{2}\right)=d_{\xi}\left(\left(\mu_{1}\left(\rho, T_{1}\right)+\mu_{2}\left(\rho, T_{2}\right)\right) d_{\xi} u\right), \\
d_{\xi}\left(\rho E+p_{1}+p_{2}\right) u=d_{\xi}\left(\left(\mu_{1}\left(\rho, T_{1}\right)+\mu_{2}\left(\rho, T_{2}\right)\right) u d_{\xi} u\right), \\
d_{\xi} p_{2} u+\left(\gamma_{2}-1\right) p_{2} d_{\xi} u=\left(\gamma_{2}-1\right) \mu_{2}\left(\rho, T_{2}\right)\left(d_{\xi} u\right)^{2}-\left(\gamma_{2}-1\right) \rho \epsilon\left(T_{2}\right) .
\end{array}\right.
$$

The first equation in (3.6) implies that the relative mass flux $\rho u$ has a constant value denoted by $m=(\rho u)_{L}$. Arguing again about the Galilean invariance allows us to assume without loss of generality that:

$$
m>0 .
$$

In other words, we only address from now on viscous profiles for shock solutions associated with the first GNL field according to the Lax condition stated in Theorem 2.1, namely we restrict attention to a left state $\mathbf{v}_{L} \in \Omega$ such that (see also $[4,14]$ )

$$
m>(\rho c)_{L}, \quad c_{L}=\sqrt{\frac{\gamma_{1}\left(p_{1}\right)_{L}}{\rho_{L}}} .
$$


Using the identity $u=m \tau$ with $\tau=1 / \rho$ and invoking Lemma 3.1, smooth solutions of (3.6) must equivalently solve the following autonomous system. We denote $\Sigma^{3}$ to shorten the notations:

$$
\left\{\begin{array}{l}
m d_{\xi} \tau=\frac{m^{2}\left(\tau-\tau_{L}\right)+s_{1} \tau^{-\gamma_{1}}+s_{2} \tau^{-\gamma_{2}}-\left(s_{1} \tau^{-\gamma_{1}}+s_{2} \tau^{-\gamma_{2}}\right)_{L}}{\mu_{1}\left(\tau, s_{1}\right)+\mu_{2}\left(\tau, s_{2}\right)} \equiv g\left(\tau, s_{1}, s_{2}\right), \\
m d_{\xi} s_{1}=\left(\gamma_{1}-1\right) \tau^{\gamma_{1}-1}\left(\mu_{1}\left(\tau, s_{1}\right) g^{2}\left(\tau, s_{1}, s_{2}\right)+\frac{\epsilon\left(\tau, s_{2}\right)}{\tau}\right), \\
m d_{\xi} s_{2}=\left(\gamma_{2}-1\right) \tau^{\gamma_{2}-1}\left(\mu_{2}\left(\tau, s_{2}\right) g^{2}\left(\tau, s_{1}, s_{2}\right)-\frac{\epsilon\left(\tau, s_{2}\right)}{\tau}\right),
\end{array}\right.
$$

equipped with the natural phase space:

$$
\mathcal{E}\left(\Sigma^{3}\right)=\left\{\omega=\left(\tau, s_{1}, s_{2}\right) \in \mathbb{R}^{3} / \tau>0, s_{1}>0, s_{2} \geq 0\right\} .
$$

In (3.9) and in the sequel, we set with little abuse in the notations, $\mu_{i}\left(\tau, s_{i}\right)=$ $\mu_{i}\left(\rho, T_{i}\left(\rho, s_{i}\right)\right)$ for $i=1,2$ and $\epsilon\left(\tau, s_{2}\right)=\epsilon\left(T_{2}\left(\rho, s_{2}\right)\right)$. In view of condition (2.5), note that $\mu_{1}\left(\tau, s_{1}\right)+\mu_{2}\left(\tau, s_{2}\right)>0$ over $\mathcal{E}\left(\Sigma^{3}\right)$. Then the smoothness assumptions made on the viscosity laws clearly show the vector field $X: \mathcal{E}\left(\Sigma^{3}\right) \rightarrow \mathbb{R}^{3}$ to be generically continuous but only Lipschitz continuous (actually differentiable) for turbulent viscosity satisfying (2.6). Well-known results then assert that prescribing at $\xi=0$ an initial data $\omega_{0}$ in (3.9) gives rise to a non-extensible solution of $\Sigma^{3}$ under the generic assumption of a continuous vector field $X$ (see Reinhard [12] or Walter [15] for instance), uniqueness being achieved under the strengthened condition of a Lipschitz continuous field (see again $[12,15]$ for the Picard-Lindelöf Theorem and for counterexamples).

3.2. A reduced dynamical system. The reduced form of the dynamical system $\Sigma^{3}$ will follow from the next statement:

Lemma 3.2. The non extensible solutions of $\Sigma^{3}$, with $\lim _{\xi \rightarrow-\infty} \omega(\xi)=\omega_{L}$, equivalently solve the next algebraic-differential system:

$$
\left\{\begin{array}{l}
m d_{\xi} \tau=\frac{\mathcal{F}(\tau)+\frac{\gamma_{2}-\gamma_{1}}{\gamma_{2}-1} s_{2} \tau^{1-\gamma_{2}}}{\tau\left(\mu_{1}\left(\tau, s_{1}\right)+\mu_{2}\left(\tau, s_{2}\right)\right)} \equiv \mathcal{G}\left(\tau, s_{1}, s_{2}\right) \\
m d_{\xi} s_{2}=\left(\gamma_{2}-1\right) \tau^{\gamma_{2}-1}\left(\mu_{2}\left(\tau, s_{2}\right) \mathcal{G}^{2}\left(\tau, s_{1}, s_{2}\right)-\frac{1}{\tau} \epsilon\left(\tau, s_{2}\right)\right) \\
\frac{s_{1}(\xi) \tau(\xi)^{-\gamma_{1}}}{\gamma_{1}-1}+\frac{s_{2}(\xi) \tau(\xi)^{-\gamma_{2}}}{\gamma_{2}-1}= \\
\frac{\tau_{L}}{\tau(\xi)} \frac{\left(p_{1}\right)_{L}}{\gamma_{1}-1}+\left(1-\frac{\tau_{L}}{\tau(\xi)}\right)\left(\frac{m^{2}}{2}\left(\tau(\xi)-\tau_{L}\right)-\left(p_{1}\right)_{L}\right)
\end{array}\right.
$$

where we have set

$$
\begin{aligned}
& \mathcal{F}(\tau)=m^{2}\left(\frac{\gamma_{1}+1}{2}\right)\left(\tau-\tau_{L}\right)\left(\tau-\tau^{\star}\left(\mathbf{v}_{L}\right)\right), \\
& \tau^{\star}\left(\mathbf{v}_{L}\right)=\frac{\gamma_{1}-1}{\gamma_{1}+1} \tau_{L}+\frac{2 \gamma_{1}}{\gamma_{1}+1} \frac{\left(p_{1}\right)_{L}}{m^{2}}
\end{aligned}
$$

The proof is postponed to the end of this section. Let us put forward an important consequence of the reported equivalence. Observe that solutions of $\Sigma^{3}$ with initial data $\omega_{0}$ in $\mathcal{E}\left(\Sigma^{3}\right)$ satisfying $\tau(\xi)>0$ and $s_{2}(\xi) \geq 0$ for $\xi \in\left[0, \xi_{0}\right)$, for some given $\xi_{0}$, 
automatically obey $s_{1}(\xi)>0$ for all the $\xi$ under consideration. This positivity property simply follows from the estimate $d_{\xi} s_{1}>0, \xi \in\left[0, \xi_{0}\right)$, inferred from $\epsilon\left(\tau, s_{2}\right) \geq 0$ in (3.9). Hence with $\omega_{0} \in \mathcal{E}\left(\Sigma^{3}\right)$, nonextensible solutions of $\Sigma^{3}$ are such that $\tau(\xi)>0, s_{2}(\xi) \geq 0$ for all $\xi \in\left(0, \xi^{+}\left(\omega_{0}\right)\right)$ where $\xi^{+}\left(\omega_{0}\right)$ denotes the maximal positive time of existence.

Next, the algebraic relation in (3.11) obviously yields $s_{1}$ as a function of $\tau(\xi)$ and $s_{2}(\xi)$ so that the ODEs in (3.11) just depend on $\tau$ and $s_{2}$. Thanks to the equivalence of (3.9) and (3.11), we have $s_{1}\left(\tau(\xi), s_{2}(\xi)\right)>0$ so that non extensible solutions of the Cauchy problem for (3.11) with initial data in $\mathcal{E}\left(\Sigma^{3}\right)$ are such that $\tau(\xi)$ and $s_{2}(\xi)$ with $\xi \in\left[0, \xi^{+}\left(\omega_{0}\right)\right)$ belong to

$$
\mathcal{E}\left(\Sigma^{2}\right)=\left\{\left(\tau, s_{2}\right) \in \mathbb{R}^{2} / \tau>0, s_{2} \geq 0\right\}
$$

It thus makes sense to consider the reduced dynamical system $\Sigma^{2}$ over the phase space (3.14):

$$
\left\{\begin{array}{l}
m d_{\xi} \tau=\mathcal{G}\left(\tau, s_{2}\right), \\
m d_{\xi} s_{2}=\left(\gamma_{2}-1\right) \tau^{\gamma_{2}-1}\left(\mu_{2}\left(\tau, s_{2}\right) \mathcal{G}^{2}\left(\tau, s_{2}\right)-\frac{1}{\tau} \epsilon\left(\tau, s_{2}\right)\right),
\end{array}\right.
$$

where we have set

$$
\mathcal{G}\left(\tau, s_{2}\right)=\mathcal{G}\left(\tau, s_{1}\left(\tau, s_{2}\right), s_{2}\right)
$$

With little abuse in the notations, (3.11) and (3.15)-(3.16) will refer to the same dynamical system $\Sigma^{2}$ throughout the end of the present paper. Again for simplicity, the pair $\left(\tau, s_{2}\right) \in \mathcal{E}\left(\Sigma^{2}\right)$ will be still denoted by $\omega$.

We conclude this section when establishing Lemma 3.2.

Proof. Since the solutions of (3.9) equivalently solve (3.6), they obey

$$
d_{\xi}\left(E\left(\tau, s_{1}, s_{2}\right)+\tau\left(p_{1}\left(\tau, s_{1}\right)+p_{2}\left(\tau, s_{2}\right)\right)\right)=m d_{\xi}\left(\left(\mu_{1}+\mu_{2}\right) \tau d_{\xi} \tau\right)
$$

in view of $u=m \tau$. Integrating from $-\infty$ to $\xi$, we get:

$$
\left[E\left(\tau, s_{1}, s_{2}\right)+\tau\left(p_{1}\left(\tau, s_{1}\right)+p_{2}\left(\tau, s_{2}\right)\right)\right]_{-\infty}^{\xi}=\tau\left[m^{2} \tau+s_{1} \tau^{-\gamma_{1}}+s_{2} \tau^{-\gamma_{2}}\right]_{-\infty}^{\xi},
$$

from the governing ODE for $\tau$ in (3.9). The required algebraic relation in (3.11) follows after rearrangements in (3.17) when writing:

$$
E\left(\tau, s_{1}, s_{2}\right)+\tau\left(p_{1}\left(\tau, s_{1}\right)+p_{2}\left(\tau, s_{2}\right)\right)=\frac{m^{2} \tau^{2}}{2}+\frac{\gamma_{1} s_{1} \tau^{1-\gamma_{1}}}{\gamma_{1}-1}+\frac{\gamma_{2} s_{2} \tau^{1-\gamma_{2}}}{\gamma_{2}-1}
$$

3.3. Heteroclinic solutions of $\Sigma^{2}$. We analyse for existence heteroclinic solutions of $\Sigma^{2}$ issuing from $\left(\tau_{L}, 0\right)$ in the past and arriving at some state $\left(\tau_{R}, 0\right) \in$ $\mathcal{E}\left(\Sigma^{2}\right)$ in the future. With this respect, we first exhibit below a systematic heteroclinic solution.

In that aim, let us consider the auxiliary ODE problem:

$$
\left\{\begin{array}{l}
m d_{\xi} \tau=\frac{1}{\tau \mu_{1}(\tau)} \mathcal{F}(\tau) \\
\tau(0)=\tau_{0}
\end{array}\right.
$$


with $\mathcal{F}(\tau)$ given in $(3.12)$ and where we have set:

$$
\left\{\begin{array}{l}
\mu_{1}(\tau)=\mu_{1}\left(\tau, s_{1}(\tau)\right) \\
\frac{s_{1}(\tau) \tau^{-\gamma_{1}}}{\gamma_{1}-1}=\frac{\tau_{L}}{\tau} \frac{\left(p_{1}\right)_{L}}{\gamma_{1}-1}+\left(1-\frac{\tau_{L}}{\tau}\right)\left(\frac{m^{2}}{2}\left(\tau-\tau_{L}\right)-\left(p_{1}\right)_{L}\right) \equiv \frac{1}{\tau} \vartheta(\tau) .
\end{array}\right.
$$

It is worth noticing that (3.19) may be recovered from the algebraic equation in (3.11) when formally setting $s_{2}$ to zero. In the same way, observe from the assumption (2.3) and (2.7) that $\mu_{2}\left(\tau, s_{2}\right)$ and $\epsilon\left(\tau, s_{2}\right)$ vanish with $s_{2}$. Hence the function $s_{2}(\xi)=0, \xi \in \mathbb{R}$, is a natural solution of the second ODE in (3.11) so that (3.19) is deduced from (3.11) with again $s_{2}(\xi)=0$ for all $\xi \in \mathbb{R}$. The quadratic function $\vartheta(\tau)$ in (3.19) is easily seen to achieve its minimum at $\tau_{\min }=\tau_{L}+\left(p_{1}\right)_{L} / \mathrm{m}^{2}$ with

$$
\vartheta\left(\tau_{\min }\right)=\frac{\left(p_{1}\right)_{L}}{m^{2}}\left(\frac{m^{2} \tau_{L}}{\gamma_{1}-1}-\frac{\left(p_{1}\right)_{L}}{2}\right)>\frac{\gamma_{1}+1}{2\left(\gamma_{1}-1\right)}\left(\frac{\left(p_{1}\right)_{L}}{m}\right)^{2}>0,
$$

since $m^{2} \tau_{L}>\gamma_{1}\left(p_{1}\right)_{L}$ in view of the Lax condition (3.8) and $\gamma_{1}>1$. Consequently, the function $\tau \in \mathbb{R}_{\star}^{+} \rightarrow s_{1}(\tau)$ keeps strictly positive values and is clearly Lipschitz continuous. Thus $\mu_{1}\left(\tau, s_{1}(\tau)\right)$ and next the right hand side of (3.18) are well-defined and Lipschitz continuous in the reported domain (see indeed the smoothness assumption (2.6)).

Next, from (3.13), observe that the Lax condition (3.8) readily implies

$$
\tau_{L}-\tau^{\star}\left(\mathbf{v}_{L}\right)=\frac{2 \tau_{L}}{\gamma_{1}+1}\left(1-\frac{c_{L}^{2}}{u_{L}^{2}}\right)>0,
$$

so that $\tau_{L}>\tau^{\star}\left(\mathbf{v}_{L}\right)$ and $\mathcal{F}(\tau)<0$ for all $\tau \in\left(\tau^{\star}\left(\mathbf{v}_{L}\right), \tau_{L}\right)$. Hence, for any given $\tau_{0}$ in $\left(\tau^{\star}\left(\mathbf{v}_{L}\right), \tau_{L}\right)$, the problem (3.18) admits a unique time decreasing solution $\tau^{\mathcal{N} \mathcal{S}}$ connecting $\tau_{L}$ in the past and $\tau^{\star}\left(\mathbf{v}_{L}\right)$ in the future. Let us notice that $\omega^{\mathcal{N S}}$ is nothing but a traveling wave solution of the usual $3 \times 3$ Navier-Stokes equations for the pressure law $p_{1}$ (hence the notation). As a consequence, we state:

Lemma 3.3. Assuming (2.7), i.e. $\mu_{2}(\tau, 0)=0$ for all $\tau>0$ and (2.3), namely $\epsilon(0)=0$, then $\omega^{\mathcal{N S}}: \xi \in \mathbb{R} \rightarrow \omega^{\mathcal{N} \mathcal{S}}(\xi) \in \mathcal{E}\left(\Sigma^{2}\right)$ defined by

$$
\omega^{\mathcal{N S}}(\xi)=\left(\tau^{\mathcal{N S}}(\xi), 0\right), \quad \xi \in \mathbb{R},
$$

is an heteroclinic solution of (3.15) issuing from $\omega_{L}=\left(\tau_{L}, 0\right)$ and arriving at

$$
\omega_{R}\left(\omega_{L}\right)=\left(\tau^{\star}\left(\mathbf{v}_{L}\right), 0\right) .
$$

The above consideration therefore guaranties the systematic existence of at least one traveling wave solution of (2.1) with the end point (2.15). The natural question we now tackle concerns the uniqueness of such a solution. Assuming smoothness in the turbulent viscosity law, we first state the following expected uniqueness result:

THEOREM 3.4. Assume that both viscosity laws $\mu_{1}$ and $\mu_{2}$ meet the condition (2.6) while the turbulent dissipation rate $\epsilon$ satisfies (2.3). Then under the Lax condition (3.8), the natural solution (3.21) is the unique heteroclinic solution of (3.11) issuing from $\omega_{L}$ and arriving at $\omega_{R}\left(\omega_{L}\right)$ given in (3.22). Traveling wave solutions are thus unique (up to some translation). 
In sharp contrast, the above uniqueness result is lost when the turbulent viscosity law in (2.1) falls within the framework of (2.8)-(2.10):

TheOREM 3.5. Suppose that $\mu_{1}$ obeys (2.6) while $\mu_{2}$ meets the assumptions (2.7)(2.10). Assume that $\epsilon$ obeys (2.11). Then under the Lax condition (3.8), there exist infinitely many heteroclinic orbits connecting the same pair of end points $\omega_{L}$ and $\omega_{R}\left(\omega_{L}\right)$ in (3.22). The system (2.1) thus admits multiple traveling solutions.

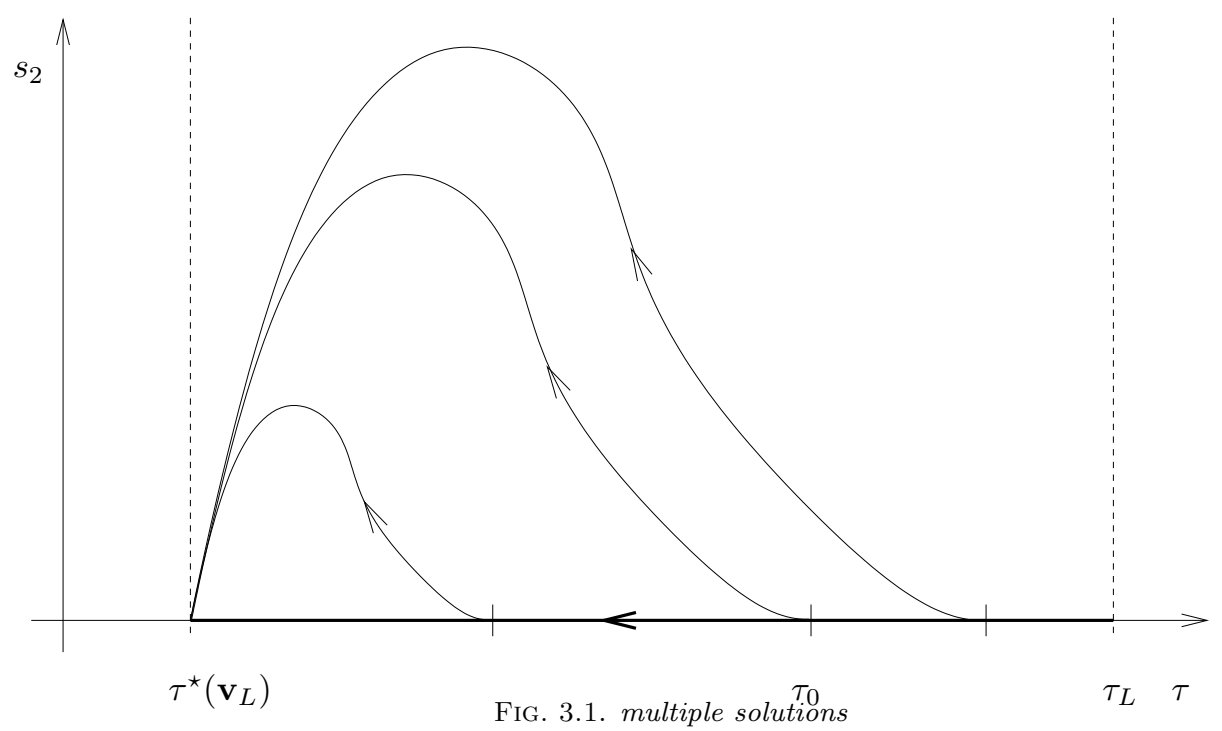

Figure 3.1 gives a physical picture of the above two results. The natural and expected solution (3.21) is depicted in bold line. Given any $\tau_{0} \in\left(\tau^{\star}\left(\mathbf{v}_{L}\right), \tau_{L}\right)$, another solution with a non-zero turbulent entropy displayed in solid line has to be considered according to Theorem 3.5 as soon as the turbulent viscosity law fails to be Lipschitz continuous. This additional solution clearly starts from $\tau_{L}$ in the past then bifurcates from a zero turbulent entropy at $\tau_{0}$ to reach in the future the expected end state $\omega_{R}\left(\omega_{L}\right)$.

The validity of the above statements will follow from the next technical results. Concerning the Theorem 3.4, the uniqueness of the heteroclinic orbit (3.21) is a clear consequence of the next technical result:

Proposition 3.6. Under the smoothness condition (2.6) and the assumptions of Theorem 3.4, there exists at most one heteroclinic solution of (3.11) connecting the state $\mathbf{v}_{L}$ in the past.

Hence, the systematic solution exhibited in Lemma 3.3 is the only one in the setting of a turbulent viscosity law satisfying (2.6).

Proof. We prove in a first step that there exists at most two negative semi-orbits of (3.15) connecting the state $\omega_{L}=\left(\tau_{L}, 0\right)$ in the past. This is a direct consequence of the Center Manifold Theorem [9] which clearly applies in view of the smoothness assumptions (2.3) and (2.6). 
Tedious but easy calculations ensure that at an equilibrium point $\omega_{e}$ of the dynamical system (3.15), necessarily of the form $\omega_{e}=\left(\tau_{e}, 0\right)$, the linearization of the vector field yields:

$$
\left(\begin{array}{cc}
\frac{\mathcal{F}^{\prime}\left(\tau_{e}\right)}{\tau_{e} \mu_{1}\left(\tau_{e}, s_{1}\left(\tau_{e}, 0\right)\right)} & \frac{\gamma_{2}-\gamma_{1}}{\gamma_{2}-1} \tau_{e}^{1-\gamma_{2}} \\
0 & 0
\end{array}\right)
$$

due to (2.7) and the property $\lim _{T_{2} \rightarrow 0^{+}} \frac{d}{d T_{2}} \epsilon\left(T_{2}\right)=0$ stated in (2.3). The associated eigenvalues respectively read $\lambda_{1}\left(\omega_{e}\right)=\frac{m^{2}\left(\gamma_{1}+1\right)}{\tau_{e} \mu_{1}}\left(\tau_{e}-\frac{\tau_{L}+\tau^{\star}\left(\mathbf{v}_{L}\right)}{2}\right)$ and $\lambda_{2}\left(\omega_{e}\right)=0$. Hence, at the equilibrium point $\omega_{L}=\left(\tau_{L}, 0\right)$, the sign of $\lambda_{1}\left(\omega_{L}\right)$ is given by the sign of $\tau_{L}-\tau^{\star}\left(\mathbf{v}_{L}\right)$. But as already observed in (3.20), the inequality $\tau^{\star}\left(\mathbf{v}_{L}\right)<\tau_{L}$ is equivalent to the Lax condition (3.8) and therefore we have under (3.8): $\lambda_{1}\left(\omega_{L}\right)>0$.

It can be seen that the associated eigenspace $T^{u}\left(\omega_{L}\right)$ is spanned by $e_{1}=(1,0)$. From the Center Manifold Theorem [9], there exists an unstable manifold, $W^{u}\left(\omega_{L}\right)$, of dimension 1 and tangent to $T^{u}\left(\omega_{L}\right)$ at $\omega_{L}$, made of the totality of the orbits which tend exponentially fast to $\omega_{L}$ as $\xi \rightarrow-\infty$. Since $W^{u}\left(\omega_{L}\right)$ is tangent to $e_{1}$ at $\omega_{L}$, there exist exactly two horizontal orbits approaching $\omega_{L}$ as $\xi \rightarrow-\infty$ from the two opposite directions $\tau \geq \tau_{L}$ and $\tau \leq \tau_{L}$.

Next, to conclude the proof, we establish that the positive semi-orbit emanating from $\tau \geq \tau_{L}$ cannot give rise to a heteroclinic solution. In that aim, let be given $\epsilon>0$, arbitrarily small, so as to define the domain

$$
\mathcal{I}_{\epsilon}=\left\{\omega \in \mathcal{E}\left(\Sigma^{2}\right) / \tau>\tau_{L}+\epsilon, s_{2} \geq 0\right\}
$$

and its left boundary

$$
\Gamma_{\epsilon}=\left\{\omega \in \mathcal{E}\left(\Sigma^{2}\right) / \tau=\tau_{L}+\epsilon, s_{2} \geq 0\right\},
$$

with unit inward normal $\mathbf{n}_{\Gamma_{\epsilon}}=(1,0)$. Then the scalar product of the vector field $X(\omega)$ of (3.15), $\omega \in \Gamma_{\epsilon}$ with $\mathbf{n}_{\Gamma_{\epsilon}}$ reads:

$$
X\left(\tau_{L}+\epsilon, s_{2}\right) \cdot \mathbf{n}_{\Gamma_{\epsilon}}=\mathcal{G}\left(\tau_{L}+\epsilon, s_{2}\right) .
$$

But under the Lax condition (3.8), we recall that $\tau^{\star}\left(\mathbf{v}_{L}\right)<\tau_{L}$ so that $\mathcal{F}(\tau)>0$ for all $\tau>\tau_{L}$ and hence $\mathcal{G}\left(\tau_{L}+\epsilon, s_{2}\right)>0$ for all $\epsilon>0$ since by $(2.2)$ we have $1<\gamma_{1}<\gamma_{2}$. As a consequence, (3.23) shows that $\Gamma_{\epsilon}$ is an entrance boundary for $\mathcal{I}_{\epsilon}$ (see [15] for instance). But the positive semi-orbit $\gamma_{+}\left(\omega_{L}\right)$ emanating from $\omega_{L}=\left(\tau_{L}, 0\right)$ with $\tau \geq \tau_{L}$ being tangent to $e_{1}$ necessarily enters $\mathcal{I}_{\epsilon}$ for any given $\epsilon$ in $\left(0, \epsilon_{0}\right), \epsilon_{0}$ small enough, and by well-known considerations then stays in $\mathcal{I}_{\epsilon}$ for all positive time. But $\mathcal{G}\left(\tau, s_{2}\right)>0$ for all states in $\mathcal{I}_{\epsilon}$ implies that there exists no critical point in $\mathcal{I}_{\epsilon}$ for all $\epsilon>0$. This completes the proof.

The proof of Theorem 3.5 follows from the next considerations.

Within the frame (2.7)-(2.10) for the viscosity law $\mu_{2}$, the existence of multiple solutions will be derived into the following three steps. In the first step, we propose to consider the following auxiliary dynamical system:

$$
\left\{\begin{aligned}
m d_{\xi} \mathcal{T}= & \frac{\mathcal{F}(\mathcal{T})+\frac{\gamma_{2}-\gamma_{1}}{\gamma_{2}-1}|S|^{\frac{1}{1-\alpha}} \mathcal{T}^{1-\gamma_{2}}}{\mathcal{T}\left(\mu_{1}+\mu_{2}\right)\left(\mathcal{T},|S|^{\frac{1}{1-\alpha}}\right)} \equiv \tilde{\mathcal{G}}(\mathcal{T}, S) \\
m d_{\xi} S= & \left(\gamma_{2}-1\right) \mathcal{T}^{\gamma_{2}-1}(1-\alpha) \times \\
& \left(\frac{\mu_{2}\left(\mathcal{T},|S|^{\frac{1}{1-\alpha}}\right)}{|S|^{\frac{\alpha}{1-\alpha}}} \tilde{\mathcal{G}}^{2}(\mathcal{T}, S)-\frac{1}{\mathcal{T}|S|^{\frac{\alpha}{1-\alpha}}} \epsilon\left(\frac{\mathcal{T}^{1-\gamma_{2}}|S|^{\frac{1}{1-\alpha}}}{\gamma_{2}-1}\right)\right),
\end{aligned}\right.
$$


where

$$
\mu_{1}\left(\mathcal{T},|S|^{\frac{1}{1-\alpha}}\right)=\mu_{1}\left(\mathcal{T},\left|S_{1}\left(\mathcal{T},|S|^{\frac{1}{1-\alpha}}\right)\right|\right)
$$

with

$$
\begin{aligned}
& \frac{S_{1}\left(\mathcal{T},|S|^{\frac{1}{1-\alpha}}\right) \mathcal{T}^{-\gamma_{1}}}{\gamma_{1}-1}+\frac{|S|^{\frac{1}{1-\alpha}} \mathcal{T}^{-\gamma_{2}}}{\gamma_{2}-1}= \\
& \frac{\tau_{L}}{\mathcal{T}} \frac{\left(p_{1}\right)_{L}}{\gamma_{1}-1}+\left(1-\frac{\tau_{L}}{\mathcal{T}}\right)\left(\frac{m^{2}}{2}\left(\mathcal{T}-\tau_{L}\right)-\left(p_{1}\right)_{L}\right),
\end{aligned}
$$

with a fixed $\alpha \in[1 / 2,1)$. This ODE system is equipped with the following phase space:

$$
\mathcal{A}=\left\{(\mathcal{T}, S) \in \mathbb{R}^{2} / \mathcal{T}>0\right\} .
$$

Before we analyse and we use this auxiliary system (3.24), let us observe from assumption $(2.8)$ that a non-constant function of the form $(\mathcal{T}(\xi), 0)$ for $\xi$ in some non-empty interval cannot provide us with a solution of the auxiliary ODE system since the property (2.8) and the asymptotic condition $\lim _{y \rightarrow 0^{+}} \epsilon(y) / y^{\alpha}=0$ stated in (2.11) imply that the vector field in $(3.24)$ evaluated at a state $(\mathcal{T}, 0)$ reads:

$$
\left(\tilde{\mathcal{G}}(\mathcal{T}, 0),\left(\gamma_{2}-1\right) \mathcal{T}^{\gamma_{2}-1}(1-\alpha) h(\mathcal{T}) \tilde{\mathcal{G}}^{2}(\mathcal{T}, 0)\right),
$$

where $\tilde{\mathcal{G}}(\mathcal{T}, 0)=\mathcal{F}(\mathcal{T}) /\left(\mathcal{T} \mu_{1}(\mathcal{T}, 0)\right) \neq 0$ except for $\mathcal{T}=\tau_{L}$ or $\mathcal{T}=\tau^{\star}\left(\mathbf{v}_{L}\right)$.

Arguing that $\alpha \in[1 / 2,1)$, we will prove hereafter that the vector field in (3.24) does obey the Lipschitz continuity condition for uniqueness in the Picard-Lindelöf Theorem. This smooth dynamical system will be seen to naturally give birth to positive semi-orbits of the original system $\Sigma^{2}$.

Proposition 3.7. Assume (3.8) so that $\tau^{\star}\left(\mathbf{v}_{L}\right)<\tau_{L}$. Then the Cauchy problem for the auxiliary system (3.24) with initial data

$$
(\mathcal{T}(0), S(0))=\left(\tau_{0}, 0\right), \quad \tau_{0} \in\left(\tau^{\star}\left(\mathbf{v}_{L}\right), \tau_{L}\right),
$$

admits a unique positive semi-orbit defined for all time $\xi \in[0,+\infty)$. All the states in the semi-orbit are such that $S(\xi)>0$ for all finite $\xi>0$ with $\lim _{\xi \rightarrow+\infty} S(\xi)=0$ while $\mathcal{T}(\xi)$ belongs to $\left(\tau^{\star}\left(\mathbf{v}_{L}\right), \tau_{L}\right)$ for all finite $\xi>0$ with $\lim _{\xi \rightarrow+\infty} \mathcal{T}(\xi)=\tau^{\star}\left(\mathbf{v}_{L}\right)$.

As a second step, let us build from the solution $(\mathcal{T}(\xi), S(\xi)), \xi \geq 0$, defined in Proposition 3.7 the function

$$
\xi: \mathbb{R}^{+} \rightarrow \omega_{+}(\xi)=\left(\tau(\xi)=\tau(\mathcal{T}(\xi)), s_{2}(\xi)=s_{2}(S(\xi))\right) \in \mathcal{E}\left(\Sigma^{2}\right),
$$

where the continuous functions $\tau(\mathcal{T})$ and $s_{2}(S)$ are defined by

$$
(\mathcal{T}, S) \in \mathcal{A}_{+}=\left\{(\mathcal{T}, S) \in \mathbb{R}^{2} / \mathcal{T}>0, S \geq 0\right\} \rightarrow\left(\tau=\mathcal{T}, s_{2}=S^{\frac{1}{1-\alpha}}\right) \in \mathcal{E}\left(\Sigma^{2}\right) .
$$

Observe that since $S(\xi)>0$ for all finite $\xi>0, \omega_{+}(\xi)$ actually belongs to $\mathcal{C}^{1}((0,+\infty))$ and solves by construction the ODE system (3.11) for all $\xi>0$ with

$$
\lim _{\xi \rightarrow 0^{+}} s_{2}(\xi)=0 \quad \text { and } \quad \lim _{\xi \rightarrow 0^{+}} \tau(\xi)=\tau_{0} .
$$


In other words, $\omega_{+}(\xi)$ is nothing but a solution in $\mathcal{C}^{0}([0,+\infty))$ of the Cauchy problem (3.11) with initial data $\left(\tau(0), s_{2}(0)\right)=\left(\tau_{0}, 0\right)$ for $\tau_{0} \in\left(\tau^{\star}\left(\mathbf{v}_{L}\right), \tau_{L}\right)$.

In addition, this solution obeys the following asymptotic behaviors:

$$
\lim _{\xi \rightarrow+\infty} \tau(\xi)=\tau^{\star}\left(\mathbf{v}_{L}\right) \quad \text { and } \quad \lim _{\xi \rightarrow+\infty} s_{2}(\xi)=0 .
$$

Equipped with this solution, defined for non-negative times $\xi$, let us next consider the function $\xi \in \mathbb{R} \rightarrow \omega(\xi) \in \mathcal{E}\left(\Sigma^{2}\right)$ defined by

$$
\omega(\xi)= \begin{cases}\left(\tau^{\mathcal{N S}}(\xi), 0\right) & \text { if } \xi<0 \\ \omega_{+}(\xi) & \text { otherwise }\end{cases}
$$

where $\tau^{\mathcal{N S}}$ is the solution of the ODE problem (3.18) with prescribed data $\tau_{0} \in$ $\left(\tau^{\star}\left(\mathbf{v}_{L}\right), \tau_{L}\right)$.

Let us check that this function $\omega$ belongs to $\mathcal{C}^{1}(\mathbb{R})$ and actually solves (3.11) for all time $\xi$. To that purpose, it suffices to notice that

$$
\lim _{\xi \rightarrow 0^{+}} m d_{\xi} \tau(\xi)=\frac{1}{\tau \mu_{1}(\tau)} \mathcal{F}(\tau),
$$

together with

$$
\lim _{\xi \rightarrow 0^{+}} d_{\xi} s_{2}(\xi)=0
$$

since $\lim _{\xi \rightarrow 0^{+}} \mu_{2}\left(\tau(\xi), s_{2}(\xi)\right)=0$ in view of the assumption $(2.7)$ and

$$
\lim _{\xi \rightarrow 0^{+}} \epsilon\left(\tau(\xi), s_{2}(\xi)\right)=0
$$

from (2.11).

Since this construction holds for any given $\tau_{0} \in\left(\tau^{\star}\left(\mathbf{v}_{L}\right), \tau_{L}\right)$, we have thus proved Theorem 3.5.

The next statement asserts that the auxiliary ODE system (3.24) enters the classical Picard-Lindelöf Theorem framework:

LEMMA 3.8. Let $\alpha \in[1 / 2,1)$ be given so that the assumptions (2.9)-(2.11) are satisfied. Then the vector field of (3.24) is Lipschitz continuous in $\mathcal{A}$.

Proof. The assumptions (2.9)-(2.10) just assert that the mappings

$$
(\mathcal{T}, z) \in \mathbb{R}_{\star}^{+} \times \mathbb{R} \rightarrow \mu_{2}\left(\mathcal{T},|z|^{\frac{1}{1-\alpha}}\right) \in \mathbb{R}^{+} \text {and }(\mathcal{T}, z) \in \mathbb{R}_{\star}^{+} \times \mathbb{R} \rightarrow \frac{\mu_{2}\left(\mathcal{T},|z|^{\frac{1}{1-\alpha}}\right)}{|z|^{\frac{\alpha}{1-\alpha}}} \in \mathbb{R}^{+}
$$

are Lipschitz continuous in $\mathcal{A}$. Next, the function $z \rightarrow|z|^{\frac{1}{1-\alpha}}$ is easily seen to belong to $\operatorname{Lip}([0,+\infty))$ for any given $\alpha$ in $(0,1)$. As a consequence, the mapping

$$
(\mathcal{T}, z):(0,+\infty) \times[0,+\infty) \rightarrow S_{1}\left(\mathcal{T},|z|^{\frac{1}{1-\alpha}}\right)
$$

defined in (3.25) is also Lipschitz continuous. At last, the Lipschitz continuity property of $y \in \mathbb{R}^{+} \rightarrow \frac{\epsilon(y)}{y^{\alpha}} \in \mathbb{R}^{+}$stated in (2.11) implies the Lipschitz continuity of the mapping $(\mathcal{T}, z) \in \mathbb{R}_{\star}^{+} \times \mathbb{R} \rightarrow \epsilon\left(\mathcal{T}^{1-\gamma_{2}}|z|^{\frac{1}{1-\alpha}}\right) /|z|^{\frac{\alpha}{1-\alpha}}$. 
The proof of Proposition 3.7 follows from the next technical result:

LEMMA 3.9. The domain

$$
\mathcal{D}=\left\{(\mathcal{T}, S) \in \mathcal{A} / \tau^{\star}\left(\mathbf{v}_{L}\right) \leq \mathcal{T} \leq \tau_{L}, S \geq 0, \tilde{\mathcal{G}}(\mathcal{T}, S) \leq 0\right)
$$

is compact and positively invariant.

Proof. Let us first prove that

$$
\Gamma^{0}=\left\{(\mathcal{T}, S) \in \mathcal{D} / \tau^{\star}\left(\mathbf{v}_{L}\right)<\mathcal{T}<\tau_{L}, S=0\right\}
$$

is an entrance boundary: namely the positive semi-flow of $(3.24)$ points into $\mathcal{D}$. To this end, it suffices [15] to observe in view of the formula (3.26) due to assumptions (2.8) and (2.11) that the scalar product of the vector field $X(\mathcal{T}, S=0)$ of $(3.24)$ with the unit inward normal to $\Gamma_{0}, \mathbf{n}_{\Gamma_{0}}(\mathcal{T}, S=0)=(0,1)$, reads

$$
X(\mathcal{T}, 0) . \mathbf{n}_{\Gamma_{0}}(\mathcal{T}, S=0)=\left(\gamma_{2}-1\right)(1-\alpha) T^{\gamma_{2}-1} h(\mathcal{T}) \tilde{\mathcal{G}}^{2}(\mathcal{T}, 0)>0
$$

since again $\tilde{\mathcal{G}}(\mathcal{T}, 0) \neq 0$ for all $\mathcal{T} \in\left(\tau^{\star}\left(\mathbf{v}_{L}\right), \tau_{L}\right)$.

Next, let us consider the well defined curve $\mathcal{T} \in\left(\tau^{\star}\left(\mathbf{v}_{L}\right), \tau_{L}\right) \rightarrow S(\mathcal{T})>0$ from $\tilde{\mathcal{G}}(\mathcal{T}, S(\mathcal{T}))=0$ :

$$
S(\mathcal{T})=\left(\frac{\gamma_{2}-\gamma_{1}}{\gamma_{2}-1}(-\mathcal{F}(\mathcal{T})) \mathcal{T}^{\gamma_{2}-1}\right)^{1-\alpha}
$$

Indeed, let us recall from the definition (3.12) that $\mathcal{F}(\mathcal{T})<0$ for $\mathcal{T} \in\left(\tau^{\star}\left(\mathbf{v}_{L}\right), \tau_{L}\right)$ (with $\tau^{\star}\left(\mathbf{v}_{L}\right)<\tau_{L}$ under the Lax condition (3.8)) while by assumption $\gamma_{2}>\gamma_{1}>1$. The representation formula (3.27) clearly ensures that the domain $\mathcal{D}$ is bounded. Let us now prove that the curve

$$
\Gamma=\left\{(\mathcal{T}, S) \in \mathcal{D} / \tau^{\star}\left(\mathbf{v}_{L}\right)<\mathcal{T}<\tau_{L}, S=S(\mathcal{T})\right\}
$$

is also an entrance boundary. In that aim, we observe that the vector

$$
\mathbf{n}_{\Gamma}(\mathcal{T})=\nabla_{(\mathcal{T}, S)} \Phi(\mathcal{T}, S(\mathcal{T}))
$$

with

$$
\Phi(\mathcal{T}, S)=\mathcal{F}(\mathcal{T})+\frac{\gamma_{2}-\gamma_{1}}{\gamma_{2}-1} S^{\frac{1}{1-\alpha}} \mathcal{T}^{1-\gamma_{2}}
$$

defines an outward vector to the boundary $\Gamma$ : by construction, for any given $\mathcal{T} \in$ $\left(\tau^{\star}\left(\mathbf{v}_{L}\right), \tau_{L}\right)$, we have $\Phi(\mathcal{T}, S)<0$ for $S<S(\mathcal{T})$ while $\Phi(\mathcal{T}, S)>0$ otherwise.

Next, the component of $\mathbf{n}_{\Gamma}(\mathcal{T})$ in the $S$ direction writes:

$$
\mathbf{n}_{\Gamma}^{S}(\mathcal{T})=\frac{1}{1-\alpha} \frac{\gamma_{2}-\gamma_{1}}{\gamma_{2}-1} \mathcal{T}^{1-\gamma_{2}} S(\mathcal{T})^{\frac{\alpha}{1-\alpha}}>0
$$

again in view of $\gamma_{2}>\gamma_{1}>1$ and $\alpha \in[1 / 2,1)$; so that

$$
X(\mathcal{T}, S(\mathcal{T})) . \mathbf{n}_{\Gamma}(\mathcal{T})=-\mathbf{n}_{\Gamma}^{S}(\mathcal{T})(1-\alpha)\left(\gamma_{2}-1\right) \frac{\mathcal{T}^{\gamma_{2}-2}}{S(\mathcal{T})^{\frac{\alpha}{1-\alpha}}} \epsilon\left(\frac{\mathcal{T}^{1-\gamma_{2}} S(\mathcal{T})^{\frac{1}{1-\alpha}}}{\gamma_{2}-1}\right)<0
$$

Hence, the required entrance property is shown. 
To conclude, it suffices to notice that $\left(\tau^{\star}\left(\mathbf{v}_{L}\right), S\left(\tau^{\star}\left(\mathbf{v}_{L}\right)\right)=0\right)$ and $\left(\tau_{L}, S\left(\tau_{L}\right)=0\right)$ are critical points of the dynamical system (3.24) so that they cannot be reached in a finite time. Hence, the compact domain $\mathcal{D}$ is positively invariant by the flow.

From the above result, the positive semi-orbit of system (3.24) with initial data $(\mathcal{T}(0), S(0))=\left(\tau_{0}, 0\right), \tau_{0}$ arbitrarily given in $\left(\tau^{\star}\left(\mathbf{v}_{L}\right), \tau_{L}\right)$, is relatively compact and well-known considerations [15] ensure that the positive limit set of the trajectory (the so-called $\varpi$-limit set) is non-empty, compact and connected. Moreover, the function $\omega \rightarrow \mathcal{T}(\omega)$, defined for all $\omega \in \mathcal{E}\left(\Sigma^{2}\right)$, is trivially a Lyapunov function [15] on $\mathcal{D}$ since $d_{\xi} \mathcal{T}<0$ on $\stackrel{\circ}{\mathcal{D}}$. Invoking the LaSalle Theorem (see [15]), the $\varpi$-limit set is included in $\{\omega \in \mathcal{D}: \tilde{\mathcal{G}}(\omega)=0\}$, namely either $\omega_{c}=\left(\tau_{L}, 0\right)$ or $\omega_{c}=\left(\tau^{\star}\left(\mathbf{v}_{L}\right), 0\right)$. Necessarily, the positive semi-orbit under consideration reaches the state $\left(\tau^{\star}\left(\mathbf{v}_{L}\right), 0\right)$ in the future. This concludes the proof of Proposition 3.7.

\section{Conclusion and applications}

In the present framework for compressible turbulent flow, the models proposed in the literature (Mohammadi-Pironneau [10] or Patel-Rodi-Scheuerer [11]) differ in the precise definition of the turbulent viscosity law. They take the general form:

$$
\mu_{2}\left(\rho, T_{2}\right)=L \rho f_{2}\left(\rho, T_{2}\right) T_{2}^{1 / 2},
$$

where $f_{2}$ is referred as to the damping function. Various expressions of $f_{2}$ are available and an exhaustive list can be found in [11]. The most popular closure reads

$$
f_{2}\left(\rho, T_{2}\right)=C_{\mu},
$$

for some positive constant $C_{\mu}>0$ which is commonly given the value 0.09. Besides, the Hoffmann [8] model promotes

$$
f_{2}\left(\rho, T_{2}\right)=C_{\mu} \exp \left(-\frac{1.75}{1+\mu T_{2}^{1 / 2}}\right) .
$$

These two models come with

$$
\lim _{T_{2} \rightarrow 0^{+}} \frac{\mu_{2}\left(\rho, T_{2}\right)}{T_{2}^{\frac{1}{2}}}=h(\rho)>0,
$$

where $h(\rho)=C_{\mu} L \rho$ in the frame of the standard model and $h(\rho)=C_{\mu} L \rho \mathrm{e}^{-1.75}$ in the Hoffman model. They are easily seen to obey (2.9) and (2.10) when choosing $\alpha=1 / 2$. Theorem 3.5 then asserts that these two models are ill posed.

By contrast, let us quote the Hassid-Poreh [6] model characterized by

$$
f_{2}\left(\rho, T_{2}\right)=C_{\mu}\left(1-\exp \left(-\mu T_{2}^{1 / 2}\right)\right)
$$

so that the associated viscosity law reads:

$$
\mu_{2}\left(\rho, T_{2}\right)=C_{\mu} L \rho T_{2}^{1 / 2}\left(1-\exp \left(-\mu T_{2}^{1 / 2}\right)\right)
$$

and is clearly Lipschitz continuous over $\mathbb{R}_{+}^{2}$. Theorem 3.4 guarantees uniqueness of the traveling wave solution under interest. 


\section{REFERENCES}

[1] C. Berthon and F. Coquel, Travelling wave solutions of a convective diffusive system with first and second order terms in nonconservation form, Hyperbolic problems: Theory, Numerics, Applications, Internat. Ser. Numer. Math., Vol. I (Zürich, 1998), Birkhäuser, Basel, 129, 47-54, 1999.

[2] C. Berthon and F. Coquel, Nonlinear projection methods for multi-entropies Navier-Stokes systems, Innovative Methods for Numerical Solutions of Partial Differential Equations, World Sci. Publishing, River Edge, NJ, 278-304, 2002.

[3] A. Forestier, J. M. Hérard and X. Louis, A Godunov type solver to compute turbulent compressible flows, C. R. A. S. Paris, 324(8), 919-926, 1997.

[4] D. Gilbarg, The existence and limit behavior of the one-dimensional shock layer, Amer. J. Math., 73, 256-274, 1951.

[5] E. Godlewski and P.-A. Raviart, Hyperbolic Systems of Conservations Laws, Appl. Math. Sci., Springer, 118, 1996.

[6] S. Hassid and M. Poreh, A turbulent energy dissipation model for flows with grag reduction, J. Fluids Eng., 100, 107-112, 1978.

[7] C. Hirsh, Numerical Computation of Internal and External Flows, Ed. Wiley and Sons, 1990.

[8] G. H. Hoffmann, Improved form of the low-Reynolds number $k-\epsilon$ turbulence model, Physics of Fluids, 18, 107-112, 1975

[9] A. Kelley, The stable, center-stable, center, center-unstable ands unstable manifolds, in Transversal Mapping and Flows, R. Abraham and J. Robbin, Eds., Benjamin, New-York, 1967.

[10] B. Mohammadi and O. Pironneau, Analysis of the K-Epsilon Turbulence Model, Research in Applied Mathematics, Masson, 1994

[11] V. C. Patel, W. Rodi and G. Scheuerer, Turbulence models for near wall and low Reynolds number lows: review, AIAA Journal, 23, 9, 1984.

[12] H. Reinhard, Differential Equations, Foundations and Applications, New York: Macmillan, Inc. XII, 1987.

[13] D. Serre, Systems of conservation laws I: Hyperbolicity, Entropies, Shock Waves, Translated from the French by I. N. Sneddon, Cambridge, 1999.

[14] H. Weyl, Shock waves in arbitrary fluids, Commun. Pure Appl. Math., 2, 103-122, 1949.

[15] W. Walter, Ordinary Differential Equations, Graduate texts in Mathematics, Springer, 182, 1998. 